\begin{tabular}{|c|l|}
\hline Title & KAP1 regulates type I interferon/STA T1-mediated IRF-1 gene expression \\
\hline Author(s) & $\begin{array}{l}\text { Kamitani, Shinya; Ohbay ashi, Norihiko; Ikeda, Osamu; Togi, Sumi hito; Muromoto, Ryuta; Sekine, Y uichi; Ohta, } \\
\text { Kazuhide; Ishiyama, Hironobu; Matsuda, Tadashi }\end{array}$ \\
\hline Citation & $\begin{array}{l}\text { Biochemical and Biophysical Research Communications, 370(2), 366-370 } \\
\text { https://doi.org/10.1016/.bbrc.2008.03.104 }\end{array}$ \\
\hline Issue Date & 2008-05-30 \\
\hline Doc URL & http://hdl.handle.net/2115/33893 \\
\hline Type & article (author version) \\
\hline File Information & KAP1-IFN.pdf \\
\hline
\end{tabular}

Instructions for use 


\section{Title: KAP1 regulates type I interferon/STAT1-mediated IRF-1 gene expression}

Authors: Shinya Kamitani*, Norihiko Ohbayashi*, Osamu Ikeda*, Sumihito Togi*, Ryuta Muromoto*, Yuichi Sekine*, Kazuhide Ohta\#, Hironobu Ishiyama\#, and Tadashi Matsuda*

Affiliation: *Department of Immunology, Graduate School of Pharmaceutical Sciences, Hokkaido University, Kita-Ku Kita 12 Nishi 6, Sapporo 060-0812, Japan, \# Otsuka Pharmaceutical Co., Ltd., 463-10 Kagasuno, Kawauchi-cho, Tokushima 771-0192, Japan

Address for manuscript correspondence: Dr. Tadashi Matsuda, Department of Immunology, Graduate School of Pharmaceutical Sciences, Hokkaido University, Kita-Ku Kita 12 Nishi 6, Sapporo 060-0812, Japan, TEL: 81-11-706-3243, FAX: 81-11-706-4990, E-mail: tmatsuda@pharm.hokudai.ac.jp

Running title: Interactions between STAT1 and KAP1 


\begin{abstract}
Signal transducers and activators of transcription (STATs) mediate cell proliferation, differentiation, and survival in immune responses, hematopoiesis, neurogenesis, and other biological processes. Recently, we showed that KAP1 is a novel STAT-binding partner that regulates STAT3-mediated transactivation. KAP1 is a universal corepressor protein for the KRAB zinc finger protein superfamily of transcriptional repressors. In this study, we found KAP1-dependent repression of interferon (IFN) /STAT1-mediated signaling. We also demonstrated that endogenous KAP1 associates with endogenous STAT1 in vivo. Importantly, a small-interfering RNA-mediated reduction in KAP1 expression enhanced IFN-induced STAT1-dependent IRF-1 gene expression. These results indicate that KAP1 may act as an endogenous regulator of the IFN/STAT1 signaling pathway.
\end{abstract}

Keywords: IFN; STAT1; IRF-1; KAP1; transcriptional regulation 


\section{Introduction}

The intracellular processing of cytokine signals entails the activation and nuclear translocation of STAT family transcription factors [1-3]. STATs are unusual among transcription factors in that they have characteristics of cytoplasmic signaling molecules, such as a Src-homology 2 (SH2) domain and tyrosine phosphorylation sites. Upon tyrosine phosphorylation, STATs dimerize through their phosphorylated SH2 domains and translocate to the nucleus. In recent years, constitutive or dysregulated expression of STATs has been found in cancer cells and oncogenetransfected cells and shown to be involved in a wide range of diseases. Therefore, STAT activation is tightly regulated by a variety of mechanisms.

Type I interferons (IFNs) are pleiotropic cytokines that possess several biological activities and play a central role in basic and applied research as mediators of antiviral and antigrowth responses, modulators of the immune system, and therapeutic agents against viral diseases and cancer $[4,5]$. Treatment of cells with IFN induces the activation of Jak-STAT molecules including Jak1 and Tyk2 as well as STAT1 and STAT2. Previous experiments using knock out mice have revealed that the Jak1-STAT1, STAT2 signals are major pathways for the IFN-mediated functions [6-8]. IFNactivated Jak/STAT signaling results in the transcriptional induction of many target genes, which include the genes for dsRNA-activated serine/threonine protein kinase, 2',5'-oligoadenylate synthetase and the interferon-regulatory factor (IRF) family proteins [9-11].

In attempt to identify novel STAT binding partners, we screened a mouse embryo cDNA library 
with a yeast two-hybrid system using the C-terminal domain of STAT4 as bait. We recently identifed KAP1 [12], a co-repressor of the Kruppel-associated box (KRAB)-domain-containing zinc finger proteins [13-15] as a protein that interacts specifically with STAT1, STAT3, STAT4 and STAT6. KAP1 can recruit and coordinate many components involved in gene silencing. KAP1mediated gene silencing involves the recruitment of the histone deacetylase (HDAC) complex [1618], and binding to a histone methyltransferase [19]. Therefore, KAP1 orchestrates the function of these co-repressor complexes to inhibit the transcription of its target genes.

In the present study, we show a reduction in the level of endogenous KAP1 expression in HeLa cells resulting in enhanced IFN/STAT1-mediated transcriptional activation and gene expression of IFR-1. We also demonstrate that endogenous KAP1 interacts with STAT1 in vivo. Furthermore, a combination of KAP1 and HDAC1 knock-down demonstrated that HDAC1 cooperatively acts with KAP1 in repression of IFN/STAT1-induced IRF-1-LUC activation. Therefore, these results indicate that KAP1 is an endogenous transcriptional regulator of IFN/STAT1-mediated signaling. 


\section{Materials and methods}

Reagents and antibodies.

Recombinant human IFN- $\square$ was kindly provided from Dainippon Sumitomo Pharma Company (Osaka, Japan). Expression vectors for STAT1, KAP1 and IRF-1-LUC were kindly provided by J. N. Ihle (St. Jude CRH, Memphis, TN), H. Ariga (Hokkaido Univ., Sapporo, Japan) and T. Tanaka (RCAI, RIKEN, Yokohama, Japan) [18, 20]. Anti-STAT1, anti-KAP1, anti-HDAC1, anti-HDAC2 and anti-HDAC3 antibodies were obtained from Santa Cruz Biotechnology (Santa Cruz, CA); antiHA antibody from Sigma-Aldrich (St. Louis, MO); anti-pSTAT1(Tyr705) and antipSTAT1(Ser727) antibodies from Cell Signaling Technologies (Beverly, MA); anti-Actin antibody from Chemicon International (Temecula, CA).

Cell culture, transfection, small interfering RNA (siRNA) and luciferase assays.

Human cervix carcinoma cell line HeLa and human embryonic kidney carcinoma cell line 293T were maintained in DMEM containing $10 \%$ FCS. HeLa cells were transfected using jetPEI (PolyPlus-transfection, Strasbourg, France) according to the manufacturer's instruction. 293T cells were transfected with the standard calcium precipitation protocol [21]. siRNAs targeting KAP1 and control siRNA used in this study was described previously [22]. siRNAs targeting human HDAC1, HDAC2 and HDAC3 used in this study were as follows: HDAC1, 5'GCUUCAAUCUAACUAUCAATT-3'; HDAC2, 5'-CAGUGAUGAGUAUAUCAAATT-3'; HDAC3, 5'-GCCGGUUAUCAACCAGGUATT-3'. The cells were then transfected with IRF- 
1-LUC using jetPEI. HeLa cells were plated on a 24-well plate at $2 \times 10^{4}$ cells/well, and then incubated with an siRNA-Lipofectamine 2000 (Invitrogen, Carlsbad, CA) mixture at $37^{\circ} \mathrm{C}$ for 4 hrs, followed by addition of fresh medium containing 10\% FCS. Twenty-four hrs after transfection, the cells were harvested and assayed for the luciferase activity using the Dual-Luciferase Reporter Assay System (Promega, Madison, WI) according to the manufacturer's instructions. Three or more independent experiments were carried out for each assay.

\section{Immunoprecipitation and immunoblotting.}

The immunoprecipitation and Western blotting assays were performed as described previously [21]. The immunoprecipitates from cell lysates were resolved on SDS-PAGE and transferred to PVDF transfer membrane (PerkinElmer; Boston, MA). The filters were then immunoblotted with each antibody. Immunoreactive proteins were visualized using an enhanced chemiluminescence detection system (Millipore; Bedford, MA).

$R N A$ isolation and quantitative real-time PCR.

Cells were harvested and total RNAs were prepared by using Iso-Gen (Nippon Gene, Tokyo, Japan) and used in RT-PCR. RT-PCR was performed using RT-PCR high -Plus- Kit (TOYOBO, Tokyo, Japan). Quantitative real-time PCR analyses of IRF-1, Mx1 as well as the control G3PDH mRNA transcripts were carried out using the assay-on-demandTM gene-specific fluorescently labeled TaqMan MGB probe in an ABI Prism 7000 sequence detection system (Applied Biosystems) [22].

Statistical Analysis. 
The significance of differences between group means was determined by Student's $t$-test. 


\section{Results and Discussion}

\section{$K A P 1$ regulates IFN-induced gene expression and transcriptional activation}

In our previous work, we have shown that KAP1 negatively regulates IFN-induced transcription by a transient reporter assay using the IFN-stimulated responsive element (ISRE)-LUC [12]. We further confirmed that KAP1 affects IFN-induced transcriptional activation and gene expression using siRNA to reduce the endogenous expression of KAP1 in HeLa cells. A specific siRNA for KAP1 or a control siRNA was transfected into HeLa cells and aliquots of cell lysates and total RNAs were analyzed by Western blotting and RT-PCR, respectively, to confirm reduced expression of KAP1 (Fig. 1A and B). The IFN-mediated transcriptional responses were measured using IRF-1-LUC, in which the endogenous IRF-1 promoter drives expression of the LUC gene. As shown in Fig. 1A, siRNA-mediated reduced expression of KAP1 resulted in a significant enhancement of IFN-induced IRF-1-LUC activation, strongly indicating that KAP1 negatively regulates IFN-mediated transcriptional activation in HeLa cells. To further clarify the physiological significance of the KAP1's effect on IFN-induced transcription, we examined the effect of KAP1 on IFN-induced endogenous gene expression using quantitative real-time PCR. As shown in Fig. 1B and C, IFN-induced IRF-1 and Mx1 mRNA expressions were enhanced in KAP1 siRNA-treated HeLa cells. Furthermore, a marked effect of KAP1 was seen in terms of IRF-1 mRNA expression compared with that of Mx1. As shown in Fig. 1C, IFN-induced IRF-1 and Mx1 mRNA expressions were enhanced in KAP1 siRNA-treated HeLa cells. Importantly, a 
significant up-regulation of both mRNA expressions by KAP1 siRNA treatment was observed in the immediate-early phase following IFN stimulation. These results indicate that KAP1 effectively influences the immediate-early phase of transcriptional activation in some IFN-induced genes.

KAP1 physically interacts with STAT1 in vivo

Our previous study revealed that the C-terminal region of KAP1 (amino acids 394-835) interacts with ectopically expressed STAT1, STAT3, STAT4 or STAT6, but not with STAT2, STAT5a or STAT5b in 293T cells [12]. Here, we investigated whether wild-type STAT1 interacts with KAP1 and STAT1 phosphorylation state is involved in their interactions. Expression vectors encoding HA-tagged KAP1 and STAT1 or STAT1 YF mutant were transiently transfected into 293T cells. STAT1 YF failed to be phosphorylated at Tyr705. Transfected 293T cells were lysed and subjected to immunoprecipitation with anti-HA antibody. Immunoprecipitates were then used in Western blot analysis with anti-STAT1 antibody. As shown in Fig. 2A, STAT1 YF showed significant binding to KAP1, although STAT1 WT only weakly bound to KAP1, indicating that unphosphorylated STAT1 has better affinity for KAP1. These results were similar with STAT3. Therefore, KAP1 appears to trap dephosphorylated STAT proteins to regulate STAT-mediated transactivation. To further confirm that endogenous KAP1 interacts with STAT1 in vivo, coimmunoprecipitation experiments were performed using cell extracts obtained from HeLa cells, in 
which both proteins could be detected using specific antibodies. An anti-KAP1 antibody immunoprecipitated STAT1 proteins, indicating that the binding of KAP1 to STAT1 occurs at physiological expression levels (Fig. 2B).

HDACs are involved in KAPI-mediated repression of IFN/STAT1-induced transactivation

To understand the molecular mechanisms responsible for KAP1-mediated repression of STAT1 activation, we examined whether reduction of KAP1 expression affects the phosphorylation state of STAT1 after IFN stimulation. As shown in Fig. 3A and B, we detected IFN-induced tyrosine (Tyr705)- and serine (Ser727)-phosphorylation of STAT1 in control siRNA- and KAP1 siRNAtreated HeLa cells; however, we observed no significant effect on these functions owing to a reduction in KAP1 expression in HeLa cells, although it might be hard to estimate the serine phosphorylation state of STAT1 in control siRNA- and KAP1 siRNA-treated HeLa cells since STAT1 is spontaneously phosphorylated at Ser727 in the absence of IFN treatment. Interactions of KAP1 with HDACs are known to underlie the transcriptional repression induced by KAP1 [1618]. STAT3 has been also demonstrated to associate with HDAC3, and trichostatin $\mathrm{A}$, an HDAC inhibitor, restores its transcriptional activity [23]. We also showed that KAP1 effectively bridges STAT3 and HDAC3 [12]. Recently, KAP1 was also reported to recruit HDAC to E2F1 and cause inhibition of E2F1 activity [24]. To examine whether HDACs are involved in KAP1-mediated repression of IFN/STAT1-induced IRF-1-LUC activation, we first tested the effect of HDAC 
knockdown in HeLa cells. As shown in Fig. 3C, reductions in the levels of HDAC1, HDAC2 or HDAC3 expression in HeLa cells resulted in an enhanced IFN/STAT1-induced IRF-1-LUC activation, indicating that HDACs are involved in the regulation of IFN/STAT1-induced IRF-1LUC activation. We finally examined the effect of combination knockdown of HDAC1 and KAP1 on IFN/STAT1-induced IRF-1-LUC activation in HeLa cells. As shown in Fig. 3D, a marked enhancement of IFN/STAT1-induced IRF-1-LUC activation was observed in HDAC1and KAP1-knocked down HeLa cells. These results indicate that HDAC1 cooperatively acts with KAP1 in the repression of IFN/STAT1-induced IRF-1-LUC activation in HeLa cells.

\section{Concluding remarks}

In this study, we have shown that endogenous KAP1 interacts with STAT1 and regulates IFN/STAT1-mediated IRF-1 gene expression in collaboration with HDACs. We previously demonstrated that KAP1 interacts with STAT3 and regulates an IL-6 family cytokine-mediated signaling. Taken together, these results suggest that KAP1 may act as a general transcriptional repressor for a variety of cytokine signal transduction pathways. However, as shown in Fig. 1B and C, a marked effect of KAP1 was observed in terms of IRF-1 gene expression compared with that of Mx1 and its effect was seen at the immediate-early phase after IFN stimulation, suggesting that KAP1 possesses a temporal and spatial effect on specific gene expressions. Importantly, we have recently found that KAP1 positively regulates some cytokine expressions (data not shown), 
indicating that KAP1 may act as a coactivator for certain transcription factors, although further work will be required to determine a KAP1's novel function.

Recent studies have demonstrated that a reduction in the level of KAP1 increases E2F1 activity and E2F1-mediated apoptosis after DNA damage in p53-deficient cells, suggesting that KAP1 is a novel regulator of E2F1, independent of pRB and p53 [24]. Furthermore, many studies have indicated that STAT1 possesses tumor suppressor activity, in that more rapid tumor progression has been observed in STAT1-deficient mice [25]. Thus, these proteins are involved in both oncogenic and tumor suppressor functions, suggesting that KAP1 may be deeply involved in the development of cancer. More interestingly, a KAP1 corepressor complex was recently demonstrated to be involved in epigenetic silencing of retrovirus transcription in embryonic cells [26], suggesting that KAP1 binds to the retrovirus primer binding site and plays an important role in the embryonic host defense system against viral infection. Several studies demonstrated that viruses utilize IRF family protein to ensure their replication, disrupting host defense mechanisms. For example, human immunodeficiency virus (HIV)-1 carries an ISRE-like element in the 5' LTR. HIV-1 infection induces expression of IRF-1, which then stimulates HIV-1 transcription through this element both in the presence and absence of Tat [27]. In contrast, IRF-1 has been shown to be one of the key host factors that regulate intracellular hepatitis $\mathrm{C}$ virus $(\mathrm{HCV})$ replication through modulation of interferon-stimulated-gene-mediated antiviral responses [28]. Therefore, there might be a possibility that KAP1 is one of target proteins of HIV-1 or HCV infection, which controls IRF-1 activation. Further detailed work will be required to clarify the role of KAP1 in viral infection and 
will provide insights toward the development of a novel therapeutic strategy for the treatment of cancer and infectious diseases. 


\section{Acknowlegements}

We thank J. N. Ihle, H. Ariga and T. Tanaka for their kind gifts of reagents. We also thank S.

Nakazato and N. Kodama for technical assistance. This study was supported in part by Sankyo

Foundation of Life Science, Industrial Technology Research Grant Program in 2005 from New

Energy and Industrial Technology Development Organization (NEDO) of Japan and Grant-in-Aid for scientific research from Ministry of Education, Culture, Sports, Science and Technology of Japan. 


\section{References}

[1] J.E. Darnell Jr.., I.M. Kerr, G.R. Stark, Jak-STAT pathways and transcriptional activation in response to IFNs and other extracellular signaling proteins, Science 264 (1994) 1415-1421.

[2] J.N. Ihle, STATs: signal transducers and activators of transcription, Cell 84 (1996) 331-334.

[3] J.J. O'Shea, Jaks, STATs, cytokine signal transduction, and immunoregulation: are we there yet?, Immunity 7 (1997) 1-11.

[4] C. Bogdan, J. Mattner, U. Schleicher, The role of type I interferons in non-viral infections, Immunol. Rev. 202 (2004) 33-48.

[5] L.C. Platanias, Mechanisms of type-I- and type-II-interferon-mediated signalling, Nat. Rev. Immunol. 5 (2005) 375-386.

[6] J.E. Durbin, R. Hackenmiller, M.C. Simon, D.E. Levy, Targeted disruption of the mouse Stat1 gene results in compromised innate immunity to viral disease, Cell 84 (1996) 443-450.

[7] M.A. Meraz, J.M. White, K.C. Sheehan, E.A. Bach, S.J. Rodig, A.S. Dighe, D.H. Kaplan, J.K. Riley, A.C. Greenlund, D. Campbell, K. Carver-Moore, R.N. DuBois, R. Clark, M. Aguet, R.D. Schreiber, Targeted disruption of the Statl gene in mice reveals unexpected physiologic specificity in the JAK-STAT signaling pathway, Cell 84 (1996) 431-442.

[8] C. Park, S. Li, E. Cha, C. Schindler, Immune response in Stat2 knockout mice, Immunity 13 (2000) 795-804.

[9] J.E. Darnell, Jr., Studies of IFN-induced transcriptional activation uncover the Jak-Stat pathway, J. Interferon Cytokine Res. 18 (1998) 549-554. 
[10] G. R.Stark, I. M. Kerr, B. R. Williams, R. H. Silverman, R. D. Schreiber, 1998. How cells respond to interferons, Annu. Rev. Biochem. 67 (1998) 227-264.

[11] T. Taniguchi, K. Ogasawara, A. Takaoka, N.Tanaka, IRF family of transcription factors as regulators of host defense, Annu. Rev. Immunol. 19 (2001) 623-655.

[12] R. Tsuruma, N. Ohbayashi, S. Kamitani, O. Ikeda, N. Sato, R. Muromoto, Y. Sekine, K. Oritani, T. Matsuda, Physical and functional interactions between STAT3 and KAP1, Oncogene (2007)

[13] S.S. Kim, Y.M. Chen, E. O'Leary, R. Witzgall, M. Vidal, J.V. Bonventre, A novel member of the RING finger family, KRIP-1, associates with the KRAB-A transcriptional repressor domain of zinc finger proteins, Proc. Natl. Acad. Sci. USA 93 (1996) 15299-15304.

[14] J.R. Friedman, W.J. Fredericks, D.E. Jensen, D.W. Speicher, X.P. Huang, E.G. Neilson, F.J. Rauscher 3rd, KAP-1, a novel corepressor for the highly conserved KRAB repression domain, Genes Dev. 10 (1996) 2067-2078.

[15] Y. Agata, E. Matsuda, A. Shimizu, Two novel Kruppel-associated box-containing zinc-finger proteins, KRAZ1 and KRAZ2, repress transcription through functional interaction with the corepressor KAP-1 (TIF1beta/KRIP-1), J. Biol. Chem. 274 (1999) 16412-16422.

[16] C. Underhill, M.S. Qutob, S.P. Yee, J. Torchia, A novel nuclear receptor corepressor complex, N-CoR, contains components of the mammalian SWI/SNF complex and the corepressor KAP-1, J. Biol. Chem. 275 (2000) 40463-40470.

[17] D.C. Schultz, J.R. Friedman, F.J. Rauscher 3rd, Targeting histone deacetylase complexes via 
KRAB-zinc finger proteins: the PHD and bromodomains of KAP-1 form a cooperative unit that recruits a novel isoform of the Mi-2alpha subunit of NuRD, Genes Dev. 15 (2001) 428-443.

[18] A. Satou, T. Taira, S.M. Iguchi-Ariga, H. Ariga, A novel transrepression pathway of c-Myc. Recruitment of a transcriptional corepressor complex to c-Myc by MM-1, a c-Myc-binding protein, J. Biol. Chem. 276 (2001) 46562-46567.

[19] D.C. Schultz, K. Ayyanathan, D. Negorev, G.G. Maul, F.J. Rauscher 3rd, SETDB1: a novel KAP-1-associated histone H3, lysine 9-specific methyltransferase that contributes to HP1-mediated silencing of euchromatic genes by KRAB zinc-finger proteins, Genes Dev. 16 (2002) 919-932. [20] S.H. Sims, Y. Cha, M.F. Romine, P.Q. Gao, K. Gottlieb, A.B. Deisseroth, A novel interferoninducible domain: structural and functional analysis of the human interferon regulatory factor 1 gene promoter, Mol. Cell. Biol. 13 (1993) 690-702.

[21] T. Matsuda, T. Yamamoto, A. Muraguchi, F. Saatcioglu, Cross-talk between transforming growth factor-beta and estrogen receptor signaling through Smad3, J. Biol. Chem. 276 (2001) $42908-42914$.

[22] Y. Sekine, O. Ikeda, Y. Hayakawa, S. Tsuji, S. Imoto, N. Aoki, K. Sugiyama, T. Matsuda, DUSP22/LMW-DSP2 regulates estrogen receptor-alpha-mediated signaling through dephosphorylation of Ser-118, Oncogene 26 (2007) 6038-6049.

[23] Z.L. Yuan, Y.J. Guan, D. Chatterjee, Y.E. Chin, Stat3 dimerization regulated by reversible acetylation of a single lysine residue, Science 307 (2005) 269-273.

[24] C. Wang, F.J. Rauscher 3rd, W.D. Cress, J. Chen, Regulation of E2F1 function by the nuclear 
corepressor KAP1, J. Biol. Chem. 282 (2007) 29902-29909.

[25] A. Stephanou, D.S. Latchman, STAT-1: a novel regulator of apoptosis, Int. J. Exp. Pathol. 84 (2003) 239-244.

[26] D. Wolf, S.P. Goff, TRIM28 mediates primer binding site-targeted silencing of murine leukemia virus in embryonic cells, Cell 131 (2007) 46-57.

[27] M. Sgarbanti, A. Borsetti, N. Moscufo, M.C. Bellocchi, B. Ridolfi, F. Nappi, G. Marsili G. Marziali, E.M. Coccia, B. Ensoli, A. Battistini, Modulation of human immunodeficiency virus 1 replication by interferon regulatory factors, J. Exp. Med. 195 (2002) 1359-1370.

[28] N. Kanazawa, M. Kurosaki, N. Sakamoto, N. Enomoto, Y. Itsui, T. Yamashiro, Y. Tanabe, S. Maekawa, M. Nakagawa, C.H. Chen, S. Kakinuma, S. Oshima, T. Nakamura, T. Kato, T. Wakita, M.Watanabe, Regulation of hepatitis $\mathrm{C}$ virus replication by interferon regulatory factor 1, J. Virol. 78 (2004) 9713-9720. 


\section{Figure legends}

Figure 1. KAP1 regulates IFN-induced gene expression and transcriptional activation

(A) HeLa cells in a 24-well plate were transfected with control siRNA or siRNA targeting human KAP1 using Lipofectamine2000. The cells were then transfected with IRF-1-LUC using jetPEI. At $36 \mathrm{~h}$ after transfection, cells were treated with IFN- $(10,000 \mathrm{U} / \mathrm{ml})$ for an additional $8 \mathrm{~h}$. The cells were harvested and assayed for the luciferase activity using the Dual-Luciferase Reporter Assay System. The results are indicated as fold induction of luciferase activity from triplicate experiments, and the error bars represent the S.D. *, p $<0.01 \quad$ An aliquot of total cell lysates (TCL) were analyzed by immunoblotting using anti-KAP1 or anti-Actin antibody. (B) HeLa cells in a 24-well plate were treated with control or KAP1 siRNA, and cells were stimulated with IFN- $\square(1,000 \mathrm{U} / \mathrm{ml})$ for an additional $2 \mathrm{~h}$. Total RNA samples isolated from these cells were subjected to RT-PCR analysis using IRF-1, Mx1, KAP1 or G3PDH primers. (C) HeLa cells in a 24-well plate were treated with control or KAP1 siRNA, and cells were stimulated with IFN- $\square(1,000 \mathrm{U} / \mathrm{ml})$ for an additional $2 \mathrm{~h}$ or 12h. IRF-1 and Mx1 expression levels were also quantified by reverse transcription and quantitative real-time PCR analysis using the assay-on-demandTM gene-specific fluorescently labeled TaqMan MGB probe in an ABI Prism 7000 sequence detection system. Data represent the levels of IRF-1 and Mx1 mRNA normalized to that of a G3PDH internal control and are expressed relative to the 
value of control siRNA-treated samples. Results are representative of three independent experiments, and the error bars represent the S.D.

Figure 2. KAP1 physically interacts with STAT1 in vivo

(A) $293 \mathrm{~T}$ cells $\left(1 \times 10^{7}\right)$ were transfected with HA-KAP1 (10 \g) and/or STAT1 wild-type (WT) or STAT1 YF mutant $(10 \square g) . \quad$ At $48 \mathrm{~h}$ after transfection, the cells were lysed, and immunoprecipitated with anti-HA antibody and immunoblotted with anti-STAT1 antibody or anti-HA antibody. TCL

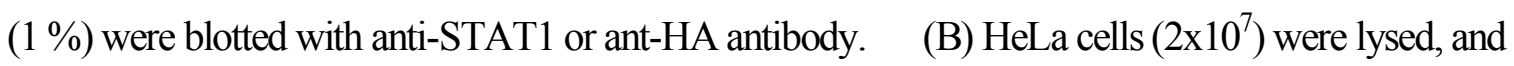
immunoprecipitated with control or anti-KAP1 antibody and immunoblotted with anti-STAT1 or antiKAP1 antibody.

Figure 3. HDACs are involved in KAP1-mediated repression of IFN/STAT1-induced transactivation

(A) HeLa cells were treated with control or KAP1 siRNA, and cells were stimulated with IFN- $\square$ $(1,000 \mathrm{U} / \mathrm{ml})$ for the indicated periods. The cells were lysed, and an aliquot of TCL was blotted with anti-pSTAT1 (Tyr705), anti-pSTAT1 (Ser727), anti-STAT1 or anti-KAP1 antibody.

Densitometric quantification of the above results was also shown. Relative intensity of pSTAT1 (Tyr705) or pSTAT1 (Ser727) was normalized to the STAT1 protein of the same sample. (C) HeLa 
cells in a 24-well plate were transfected with control, HDAC1, HDAC2 or HDAC3 siRNA, and cells were then transfected with IRF-1-LUC using jetPEI. At $36 \mathrm{~h}$ after transfection, cells were treated with IFN- $\square(10,000 \mathrm{U} / \mathrm{ml})$ for an additional $8 \mathrm{~h}$. The cells were harvested and assayed for the luciferase activity using the Dual-Luciferase Reporter Assay System. The results are indicated as fold induction of luciferase activity from triplicate experiments, and the error bars represent the S.D. $*, p<0.05 * *, p<0.01 \quad$ Total RNA samples isolated from these cells were subjected to RT-PCR analysis using HDAC1, HDAC2, HDAC3 or G3PDH primers. An aliquot of TCL was analyzed by immunoblotting using anti-HDAC1, anti-HDAC2, anti-HDAC3 or anti-Actin antibody. (C) HeLa cells in a 24-well plate were transfected with control, HDAC1 and/or KAP1 siRNA, and cells were then transfected with IRF-1-LUC using jetPEI. At $36 \mathrm{~h}$ after transfection, cells were treated with IFN- $\square(10,000 \mathrm{U} / \mathrm{ml})$ for an additional $8 \mathrm{~h}$. The cells were harvested and assayed for the luciferase activity using the Dual-Luciferase Reporter Assay System. The results are indicated as fold induction of luciferase activity from triplicate experiments, and the error bars represent the S.D. *, $\mathrm{p}<0.05 * *, \mathrm{p}<0.01$ An aliquot of TCL was analyzed by immunoblotting using anti-HDAC1, anti-KAP1 or anti-Actin antibody. 


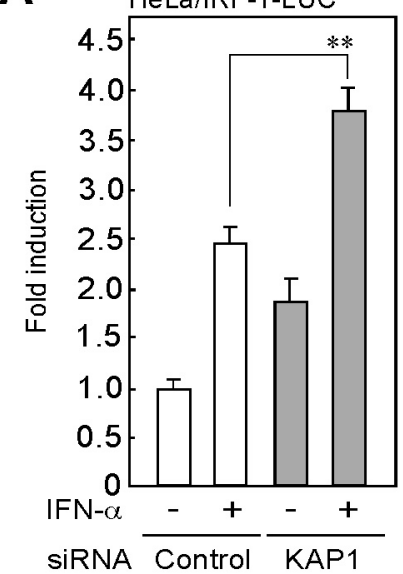

TCL

IB: KAP1

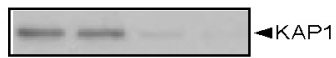

IB: Actin

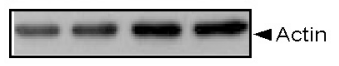

B

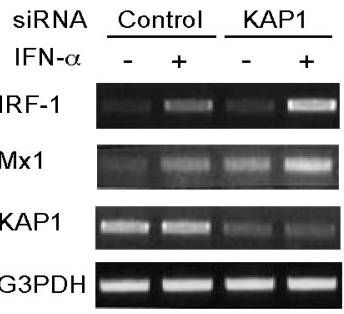

Figure 1 


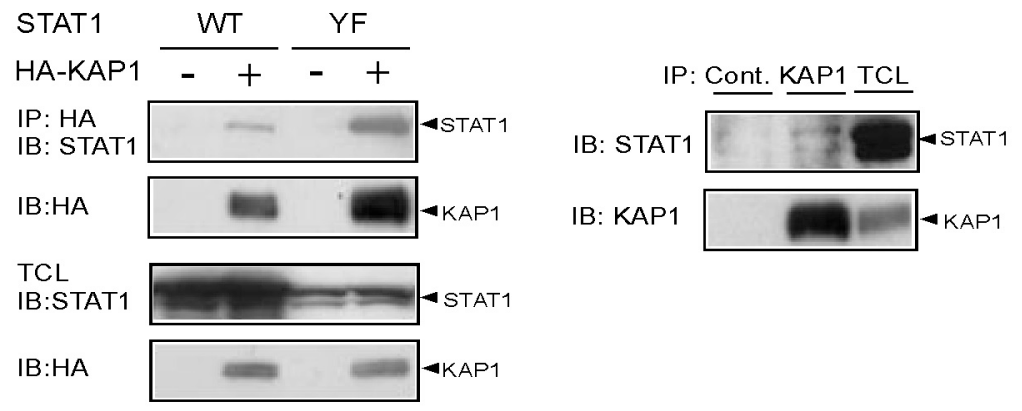


IFN- $\alpha$ stimulation TCL

IB:pSTAT1(Tyr705)

IB:pSTAT1(Ser727)

IB:STAT1

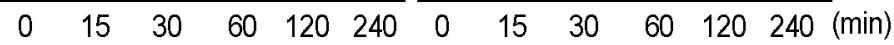

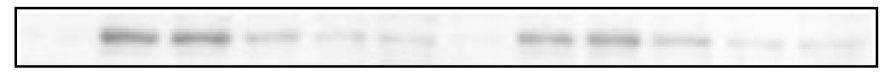

IB:KAP1

$-\cdots+\cdots+\cdots+\cdots$

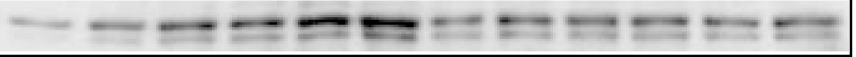

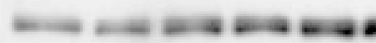
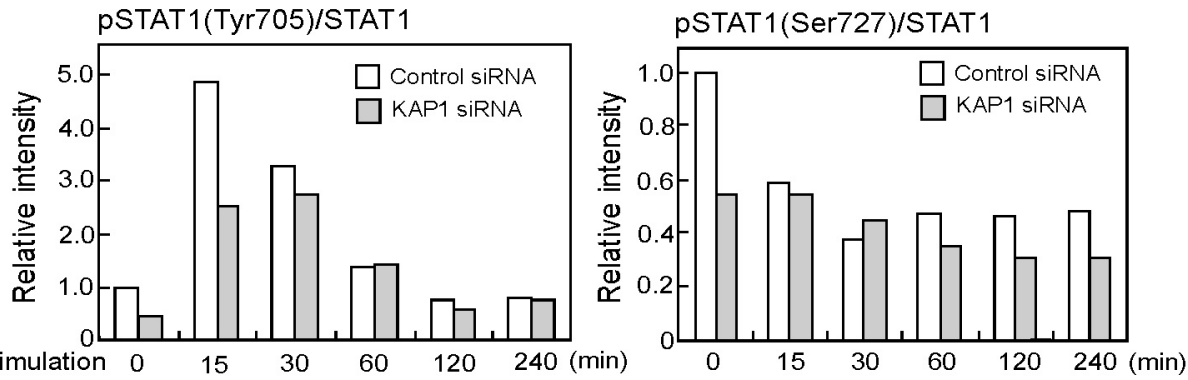

IFN- $\alpha$ stimulation

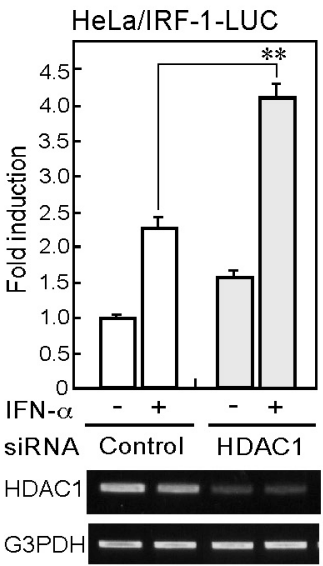

TCL

IB:HDAC1

IB:Actin
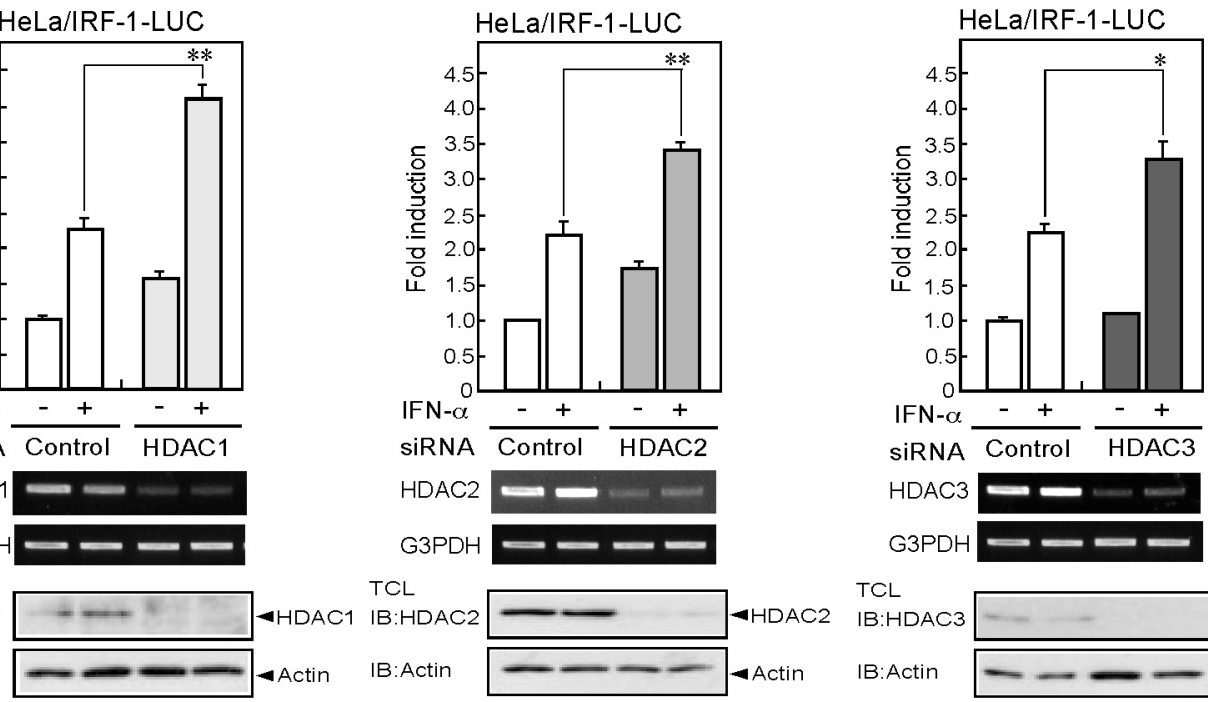

$T C L$

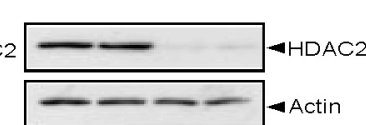

TCL

IB: HDAC3

IB: Actin

Actin

IB:Actin
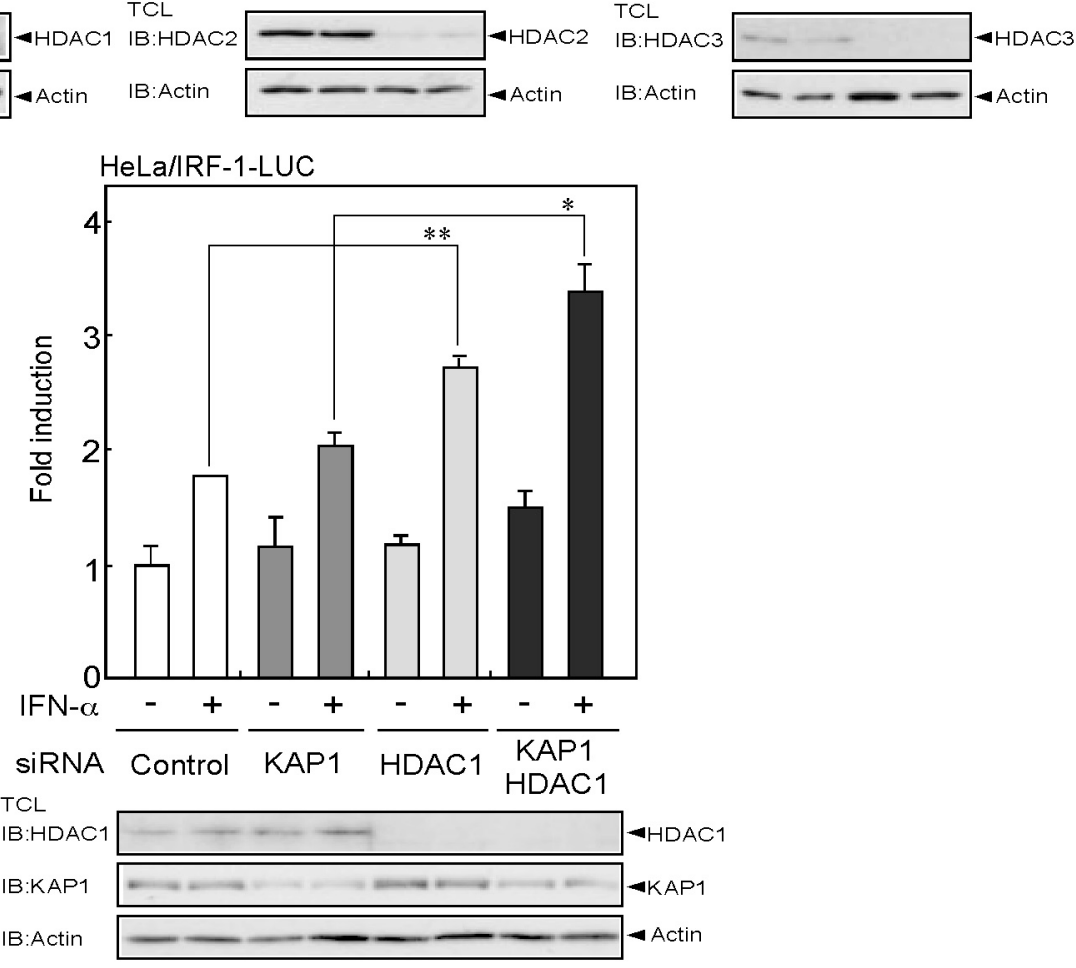

Figure 4 